\title{
THE TIME SURFACE TERM IN QUANTUM GRAVITY
}

\author{
V.N. Pervushin, V.V. Papoyan, G.A. Gogilidze, \\ A.M. Khvedelidze, Yu.G. Palii, V.I. Smirichinskii \\ Joint Institute for Nuclear Research, \\ Dubna, 141980, Moscow region, Russia \\ email: pervush@thsun1.jinr.dubna.su
}

October 9, 2018

PACS number(s):04.60.-m, 04.20.Cv, 98.80.Hw (Quantum Gravity)

\begin{abstract}
The role of the time surface term in the ADM Hamiltonian formulation of general relativity is investigated. We show that the variable contained in the time surface term (the scale factor) plays the role of a time-like variable. The conjugated variable represents the energy density in the reduced phase space, where the Schrödinger like equation for a wave function is derived. The contribution from the surface term to the phase of the wave function allows us to define the phase time of the quantum Universe so that it coincides with the proper time as an invariant interval for the classical dust filled Universe. The quantum scenario of the evolution of the Universe filled in by the WeinbergSalam fields is considered. The wave function of the early Universe as the functional from the Higgs fields and scale factor realizes the unitary irreducible representation of the $\mathrm{SO}(4,1)$ group. The elementary particle masses are determined by the angles of the scale-scalar field mixing.
\end{abstract}

\section{Statement of the problem}

The Dirac-ADM canonical approach [1, 2] to GR was the essential improvement on the way to quantization of the Einstein-Hilbert theory of gravity developed by Wheeler, DeWitt and 
others [3, 4, 5, 6]. This conventional scheme of the canonical quantization is based on the $(3+1)$ Dirac-ADM foliation [1, 2, 7, 8] of the four dimensional manifold $\left(x^{\mu}\right)$ along the some time-like vector (associated with the rest frame of an observer):

$$
d s^{2}=N^{2} d t^{2}-{ }^{(3)} g_{i k} \breve{d} x^{i} \breve{d} x^{k} \quad ; \quad\left(\breve{d} x^{i}=d x^{i}+N^{i} d t\right)
$$

(that means the restriction of the group of general coordinate transformations by the kinemetric ones [8]: $\left.t \rightarrow t^{\prime}(t) \quad ; \quad x_{i} \rightarrow x^{\prime}{ }_{i}\left(t, x_{1}, x_{2}, x_{3}\right)\right)$ and on the ADM action $\left(W_{A D M}\right)$ which differs from the initial Einstein-Hilbert action [9]

$$
W_{G R}=\int d^{4} x \sqrt{-g}\left[-\frac{{ }^{(4)} R(g)}{2 \kappa^{2}}+\mathcal{L}_{\text {matter }}\right] ; \quad\left(\kappa^{2}=8 \pi G\right),
$$

by the surface terms:

$$
W_{G R}=W_{A D M}+W_{S}+W_{T}
$$

where

$$
\begin{gathered}
W_{T}=-\int d t d^{3} x \breve{\partial}_{0}\left[\frac{\breve{\partial}_{0} \sqrt{{ }^{(3)} g}}{N \kappa^{2}}\right] ;\left[\breve{\partial}_{0} f=\dot{f}-\partial_{k}\left(N^{k} f\right)\right] . \\
W_{S}=-\int d t d^{3} x \partial_{k}\left[\sqrt{{ }^{(3)} g}\left({ }^{(3)} g^{i k} \partial_{l} N\right)\right] \frac{1}{\kappa^{2}} .
\end{gathered}
$$

For the derivation of local classical equations these surface terms are not essential, however, they play an important role in the determination of the global quantities of such a total energy [10]. It is obvious that the wave function also has global nature and depends on these surface terms. The statement of the problem consists in the canonical quantization of the Einstein-Hilbert action (1) by taking into account the surface terms (2), (3). We continue the attempts to solve this problem in the papers [11, 12.

\section{A new version of the Hamiltonian Formulation}

To solve this problem we should

I) consider the space-scale variable $a=\left[{ }^{(3)} g\right]^{\frac{1}{6}}$ in the time surface term (2) as one of dynamical variables:

$$
d s^{2}=a^{2}\left[N_{c}^{2} d t^{2}-\omega_{i \underline{k}} \omega_{j \underline{k}} \breve{d} x^{i} \breve{d} x^{j}\right], \quad ; \quad a N_{c}=N \quad ; \quad \operatorname{det} \omega=1,
$$

(we use the triad form $\omega_{i j}$ for the rest dynamic variables [13)

II) apply the Ostrogradsky method [14, 15] to the theory with second order derivative of the scale factor with respect to the time coordinate. As the result, the Hilbert action (1) in 
terms of the canonical conjugate variables $a, \pi_{a}, \Phi, \pi_{\Phi}$ (where $\Phi$ denotes the set of matter fields including graviton $\omega$ and photon $A$ ) has the form

$$
W_{G R}=\int_{0}^{T} d t \int_{V} d^{3} x\left[-\pi_{(a)} \stackrel{\odot}{a}+\frac{1}{2} \breve{\partial}_{0}\left(\pi_{(a)} a\right)+\sum_{\Phi=\omega, A} \pi_{(\Phi)} \stackrel{\odot}{\Phi}-N_{c} \mathcal{H}_{E C}\right]+W_{S},
$$

where

$$
\begin{gathered}
\stackrel{\odot}{a}=\breve{\partial}_{0} a+\frac{2}{3} a \partial_{k} N^{k}, \quad \stackrel{\odot}{A}_{k}=\partial_{0} A_{k}-\partial_{k} A_{0}-N^{l} F_{l k} \\
\dot{\odot}_{l k}=\left(\dot{\omega}_{l \underline{s}} \omega_{k \underline{s}}+\omega_{l \underline{s}} \dot{\omega}_{k \underline{s}}-\nabla_{l} N_{k}-\nabla_{k} N_{l}+\frac{2}{3} \omega_{l \underline{s}} \omega_{k \underline{s}} \partial_{j} N^{j}\right)
\end{gathered}
$$

is the kinemetric invariant time derivative (we use here covariant derivative in the metric $\omega_{i \underline{k}} \omega_{j \underline{k}}$, including the Laplace operator $\left.\Delta f=\nabla_{k} \partial^{k} f\right)$, and $\mathcal{H}_{E C}$ is the Einstein energy density

$$
\mathcal{H}_{E C}=-\frac{\kappa^{2}}{12} \pi_{(a)}^{2}+\pi_{(\omega)}^{2}\left(\frac{2 \kappa^{2}}{a^{2}}\right)+\left(\frac{a^{2}}{2 \kappa^{2}}\right) \bar{R}+\mathcal{H}_{(A)}
$$

with the photon energy

$$
\mathcal{H}_{(A)}=\frac{1}{2} \pi_{(A)}^{k} \pi_{k(A)}+\frac{1}{4} F_{i j} F^{i j}
$$

and three dimensional curvature

$$
\bar{R}=a^{2}{ }^{(3)} R\left(a^{2} \omega^{2}\right)={ }^{(3)} R\left(\omega^{2}\right)+8 a^{-\frac{1}{2}} \Delta a^{\frac{1}{2}},
$$

III) perform the canonical transformation $\left(\pi_{(a)}, a\right)=>(\Pi, \eta)$ which removes the time surface term $\frac{1}{2} \partial_{0}\left(\pi_{(a)} a\right)$

$$
\pi_{(a)} \stackrel{\odot}{a}-\frac{1}{2} \breve{\partial}_{0}\left(\pi_{(a)} a\right)=\Pi\left(\dot{\eta}-N^{k} \partial_{k} \eta\right)
$$

One can represent this transformation as

$$
\pi_{(a)}=2 \sqrt{\frac{3 \Pi}{\kappa^{2} \Gamma}} C(\eta) ; \quad a=\sqrt{\frac{\kappa^{2} \Gamma \Pi}{3}} S(\eta),
$$

where $C(\eta), S(\eta)$ and $\Gamma$ are some particular solution of the following equations

$$
C(\eta) \frac{d}{d \eta} S(\eta)-S(\eta) \frac{d}{d \eta} C(\eta)=1 ; \quad \partial_{0}(\ln \Gamma)-N^{k} \partial_{k}(\ln \Gamma)+\frac{1}{3} \partial_{k} N^{k}=0 .
$$

Finally, the Hilbert action reads in terms of the new variables as

$$
W_{G R}=\int d t d^{3} x\left[\sum_{\Phi=\omega, A, \ln \Gamma} \pi_{(\Phi)} \dot{\Phi}-\Pi \dot{\eta}-N_{c} \mathcal{H}_{E C}-N^{k} \mathcal{P}_{k}\right]+\bar{W}_{S},
$$

with space surface term $\bar{W}_{S}=W_{S}-2 \int_{0}^{T} d t d^{3} x \partial_{l}\left(N^{k} \pi_{(h)}^{l}\right)$ and constraints

$$
\begin{gathered}
\mathcal{H}_{E C}=-\frac{\Pi}{\Gamma}\left(C^{2}-S^{2} \frac{6}{\Gamma^{2}} \bar{R}\right)+\pi_{(\omega)}^{2} \frac{6}{S^{2} \Pi \Gamma}+\mathcal{H}_{(A)}=0, \\
\mathcal{P}_{k}=\pi_{(\Gamma)} \partial_{k} \ln \Gamma+\partial_{k} \pi_{(\Gamma)}+\Pi \partial_{k} \eta+2 \nabla_{l} \pi_{(\omega) k}^{l}+\pi_{(A)}^{l} F_{l k}=0 .
\end{gathered}
$$




\section{Interpretation of new variables}

To treat the new variables $\Pi$ and $\eta$, we consider the flat-space limit 12$]: \pi_{(\omega)}=\bar{R}=N^{k}=0$; $C(\eta)=1 ; S(\eta)=\eta$, where the Hilbert action (9) has the form

$$
W_{G R}=\int d t d^{3} x\left\{\pi_{(A)}^{k}\left(\dot{A}_{k}-\partial_{k} A_{0}\right)-\Pi \dot{\eta}-N_{c}\left(\mathcal{H}_{(A)}-\frac{\Pi}{\Gamma}\right)\right\} .
$$

After the reduction on the constraint shell $\mathcal{H}_{E C}=0$ in the gauge $N_{c}=1$, we get the expression for the conventional action of electrodynamics

$$
W^{\text {Red }}=\int d t d^{3} x\left\{\pi_{(A)}^{k}\left(\dot{A}_{k}-\partial_{k} A_{0}\right)-\mathcal{H}_{(A)}\right\}
$$

Note that because

$$
\dot{\eta}=\frac{1}{\Gamma}
$$

in this limit the variable $\eta$ can be treated as the time and the quantity $\Pi / \Gamma$ in the reduced phase space is a "reduced energy" like the the quantity $\sqrt{p^{2}+m^{2}}$ is the spectral energy for a relativistic particle.

\section{The wave function of the Universe}

Suppose that the constraint $\mathcal{H}_{E C}=0$ has the set of solutions $\Pi=\mathcal{H}_{(\alpha)}^{\text {red }}, \alpha=1 \ldots m$. For each solution one can write down the corresponding reduced Hilbert action

$$
W_{G R(\alpha)}^{r e d}=\int d t \int d^{3} x\left(\sum_{\Phi=\omega, A, \ln \Gamma} \pi_{(\Phi)} \dot{\Phi}-\mathcal{H}_{(\alpha)}^{r e d} \dot{\eta}\right)+\bar{W}_{S}
$$

The quantization of the action leads to the Schrödinger type evolution equation

$$
\frac{1}{i} \frac{\delta}{\delta \eta} \Psi_{\alpha}=\hat{\mathcal{H}}_{(\alpha)}^{r e d} \Psi_{\alpha}
$$

Let us consider the small time limit $(\eta) \sim 0$ which corresponds to the small Universe $(a \sim$ $S(\eta) \rightarrow 0)$. According to equation (8), in this region $S(\eta) \sim \eta, C(\eta) \sim 1$, and the graviton term $\pi_{(\omega)}^{2}$ dominates in the energy density (10). The corresponding solutions of the constraints $\mathcal{H}_{E C}=0$

$$
\Pi=\mathcal{H}_{ \pm}^{r e d}= \pm \sqrt{6 \pi_{(\omega)}^{2}} \frac{1}{\eta}
$$

and the reduced action reads

$$
W_{G R \pm}=\int_{0}^{T} d t \int_{V} d^{3} x\left(\sum \pi_{(\omega)} \dot{\omega} \mp \sqrt{6 \pi_{(\omega)}^{2}} \partial_{0} \ln \eta\right) .
$$


One can verify that in the supposition of homogeneousity of the space

$$
\begin{gathered}
d s^{2}=a^{2}\left[N_{c}^{2}(t) d t^{2}-A^{2}(r) d x^{2}\right] ; \quad A(r)=\left(1+\frac{k r^{2}}{4 r_{0}^{2}}\right)^{-1} \\
a(t) N_{c}(t) d t=d T_{\text {Fried }} ; \quad k=0, \pm 1,
\end{gathered}
$$

it follows from (16) the action for the Misner Universe [5] (in details see [6]):

$$
W_{G R \pm}=V_{(3)}\left(\pi_{(\omega)}(\omega(T)-\omega(0)) \mp \sqrt{6 \pi_{(\omega)}^{2}} \ln \frac{\eta(T)}{\eta(0)}\right),
$$

and the spectral decomposition for the wave function

$$
\Psi=\int d \pi_{(\omega)}\left(A_{\pi_{(\omega)}}^{+} e^{i W_{G R+}}+A_{\pi_{(\omega)}}^{+} e^{i W_{G R-}}\right),
$$

where the role of the time is played by the logarithm of $\eta$, and $A^{ \pm}$are the operators of creation and annihilation of the Universe. Note that in the case of the homogeneous space the particular solutions of eq. (8) read

$$
C(\eta)=1, \cos \eta, \cosh \eta ; \quad S(\eta)=\eta, \sin \eta, \sinh \eta, \quad \Gamma=r_{0}
$$

respectively for $k=0,1,-1$.

The evolution of the Universe filled in by dust and radiation has been considered in [12]. In this case the wave function of the Universe has the form $\Psi_{ \pm}=\exp \left\{\imath W_{ \pm}^{\text {red }}(a)\right\}$ with the reduced action

$$
W_{ \pm}^{r e d}= \pm V_{(3)} \int_{0}^{T} d a\left[\underline{\pi}_{(a)}-\frac{1}{2} \frac{d}{d a}\left(\underline{\pi}_{(a)} a\right)\right]
$$

where $V_{(3)}=\int d^{3} x A^{3}(r)$ and $\underline{\pi}_{(a)}=2 \sqrt{\frac{3}{\kappa^{2}}}\left[\mathcal{H}_{(M)}-\frac{3 k a^{2}}{r_{0}^{2} \kappa^{2}}\right]^{1 / 2}$ is the solution of the constraint (10) $\mathcal{H}_{E C}=0$. The phase of this function coincides (up to the energy factor) with the Friedmann time (18) for the dust case $\left(\mathcal{H}_{M}=a \epsilon_{\text {dust }}\right)$

$$
W_{d u s t}^{r e d}=\frac{V_{(3)} \epsilon_{d u s t}}{2} T_{\text {Fried }}(a) ; \quad T_{\text {Fried }}(a)=\int_{a(0)}^{a(T)} d a \frac{6 a}{\kappa^{2} \underline{\pi}_{(a)}}
$$

and with the conformal time $N_{c} d t=\eta(t) r_{0}$ for the radiation $\left(\mathcal{H}_{M}=\epsilon_{\text {rad }}\right)$

$$
W_{\text {rad }}^{r e d}=V_{(3)} \epsilon_{\text {rad }} \eta(a) r_{0} ; \quad \eta(a) r_{0}=\int_{a(0)}^{a(T)} d a \frac{6}{\kappa^{2} \underline{\pi}_{(a)}} .
$$

It is worth to note that the this clear correspondence between the quantum and classical physics ( the "phase time" and the "interval time") arises due to the maintenance of the time surface term $\frac{1}{2} \partial\left(\underline{\pi}_{(a)} a\right)$ in the Hilbert action.

Thus, the time surface term helps us to establish the correspondence between the time as the phase of the ADM wave function of the Universe and the classical proper time as an invariant interval. 


\section{Quantum scenario of the Weinberg-Salam Universe.}

Let us consider the quantum scenario of the evolution of the Universe filled in by the WeinbergSalam fields and described by the action

$$
W=\int d^{3} x d t \sqrt{-g}\left\{-\frac{{ }^{(4)} R}{2 \kappa^{2}}+\frac{{ }^{(4)} R \Phi^{*} \Phi}{6}+\partial_{\mu} \Phi^{*} \partial^{\mu} \Phi-\gamma\left(\bar{\Psi}_{L} \Phi\right) \Psi_{R}+\cdots\right\}
$$

where $\Phi=\left(\begin{array}{c}\Phi_{1} \\ \Phi_{2}\end{array}\right)$ is the doublet of the complex scalar fields; $\Psi_{L}$ and $\Psi_{R}$ are the left and right fermions. We keep only the term of the scalar-fermion interaction generating masses of the fermions $m\left(\bar{\Psi}_{L 2} \Psi_{R}+\bar{\Psi}_{R} \Psi_{L 2}\right)$ to show the evolution of the mass parameters with respect to the scale $a$. If we extract the scale factor $a$ not only from the metric (四) $g_{\mu \nu}=a^{2} \tilde{g}_{\mu \nu}$, but also from all other matter fields $\Phi=a \varphi, \quad \Psi=a^{-3 / 2} \Psi_{c}$, we can guarantee the classical limit of the massive fermion fields as the Friedmann dust of the Universe. The action in terms of the physical fields $\varphi, \Psi_{c}, \tilde{g}\left(\sqrt{-\tilde{g}}=N_{c}\right)$ has the form

$$
\begin{aligned}
W= & \int d^{4} x\left\{N _ { c } \left[-\frac{{ }^{(4)} \tilde{R}}{2 \kappa^{2}}\left(a^{2}-\frac{\kappa^{2}}{3} \varphi^{*} \varphi\right)-\frac{3}{\kappa^{2}} \partial_{\nu} a \partial^{\nu} a+\partial_{\nu} \varphi^{*} \partial^{\nu} \varphi-\gamma\left(\bar{\Psi}_{L c} \varphi^{)} \Psi_{R c}+\cdots\right]\right.\right. \\
& \left.+\partial_{\nu}\left(N_{c}\left[\frac{3 a^{2}}{\kappa^{2}}-\varphi^{*} \varphi\right] \frac{\partial^{\nu} a}{a}\right)\right\}
\end{aligned}
$$

Note that the configuration $a^{2}=\frac{\kappa^{2}}{3} \varphi^{*} \varphi$ represents the singular point: in the vicinity of this point the sign before the four- dimensional curvature is changing. So, let us consider only the field configuration such that $\left[a^{2}-\frac{\kappa^{2}}{3} \varphi^{*} \varphi\right]=\rho^{2}>0$. For this case one can introduce new variables

$$
a=\rho \cosh (\xi) ; \quad \varphi_{i}=\sqrt{\frac{3}{\kappa^{2}}} \rho \sinh (\xi) n_{i} ; \quad n_{1}=\cos (\Theta) \exp \left\{\imath \chi_{1}\right\} ; \quad n_{2}=\sin (\Theta) \exp \left\{\imath \chi_{2}\right\},
$$

where $\xi, \Theta, \chi_{1}, \chi_{2}$ are the angles of the scale-scalar mixing.

For the homogeneous space (17) with $V_{(3)}=1$ we get the action

$$
\begin{aligned}
W= & \int d t\left\{-\frac{D}{2 N_{c}}\left(\frac{6}{\kappa^{2}}\right)+N_{c}\left[\frac{k}{2 r_{0}^{2}} \rho^{2}\left(\frac{6}{\kappa^{2}}\right)-\gamma \sqrt{\left.\frac{3}{\kappa^{2}} \rho \sinh (\xi)\left(\bar{\Psi}_{L c i} n_{i}\right) \Psi_{R c}+\cdots\right]}\right.\right. \\
& \left.+\partial_{0}\left[\frac{\rho^{2}}{2 N_{c}}\left(\frac{\dot{\rho}}{\rho}+\dot{\xi} \tanh \xi\right)\right] \frac{6}{\kappa^{2}}\right\},
\end{aligned}
$$

where $D=-\dot{\rho}^{2}+\rho^{2} \dot{\xi}^{2}+(\rho \sinh \xi)^{2}\left(\dot{\Theta}^{2}+\sin ^{2}(\Theta) \dot{\chi}_{1}^{2}+\cos ^{2}(\Theta) \dot{\chi}_{2}^{2}\right)$ is the $S O(4,1)$-invariant differential form. By the Ostrogradsky method this action can be rewritten in terms of momenta

$$
W=\int d t\left\{\sum_{\alpha=\xi, \Theta, \chi_{1}, \chi_{2}} \pi_{(\alpha)} \dot{\alpha}-\pi_{(\rho)} \dot{\rho}-N_{c} \mathcal{H}_{E C}+\frac{1}{2} \partial_{0}\left(\pi_{(\rho)} \rho\right)+\frac{1}{2} \partial_{0}\left(\pi_{(\xi)} \tanh (\xi)\right)\right\}
$$




$$
\mathcal{H}_{E C}=-\frac{1}{2} \frac{\kappa^{2}}{6} \pi_{(\rho)}^{2}-\frac{k \rho^{2}}{r_{0}^{2}} \frac{6}{\kappa^{2}}+\frac{\mathcal{K}^{2}}{2 \rho^{2}} \frac{\kappa^{2}}{6}+\gamma \sqrt{\frac{3}{\kappa^{2}}} \rho \sinh (\xi)\left(\bar{\Psi}_{L c i} n_{i}\right) \Psi_{R c}+\cdots
$$

where $\mathcal{K}^{2}$ is the Kazimir operator of the $S O(4,1)$ group

$$
\mathcal{K}^{2}=P_{(\xi)}^{2}+\frac{1}{\sinh ^{2}(\xi)}\left(P_{(\Theta)}^{2}+\frac{P_{\chi_{1}}^{2}}{\sin ^{2}(\Theta)}+\frac{P_{\chi_{2}}^{2}}{\cos ^{2}(\Theta)}\right)
$$

We see that the variable $\rho$ plays the same role as the scale $a$ in the theory without scalar fields, and it is the time like variable. Note that our transition to new variables is similar to the Bekenstein transformation [18]. Repeating the canonical transformation (7) $\pi_{(\rho)} \dot{\rho}-\frac{1}{2} \partial_{0}\left(\pi_{(\rho)} \rho\right)=$ $\Pi \dot{\eta}$, where $\Gamma=r_{0}$, we get the expression for the action

$$
\begin{aligned}
W= & \int d t\left\{\sum_{\alpha} P_{(\alpha)} \dot{\alpha}-\Pi \dot{\eta}-N_{c}\left[-\frac{\Pi}{r_{0}}+\frac{\mathcal{K}^{2}}{4 \Pi r_{0} S^{2}(\eta)}+\gamma \sqrt{\frac{3}{\kappa^{2}}} \rho \sinh (\xi)\left(\bar{\Psi}_{L c i} n_{i}\right) \Psi_{R c}+\cdots\right]\right. \\
& \left.+\frac{1}{2} \partial_{0}\left(P_{(\xi)} \tanh (\xi)\right)\right\}
\end{aligned}
$$

This action describes the following ADM-scenario of the evolution of the Universe. In the small time limit $(\eta \sim 0)$ the Kazimir operator term dominates and the reduced system on the constraint $\mathcal{H}_{E C}=0$ has the form

$$
W_{( \pm)}^{r e d}=\int_{T_{s}(0)}^{T_{s}(T)} d T_{s}\left[\sum_{\alpha} P_{\alpha} \frac{d \alpha}{d T_{s}} \mp \mathcal{K}\left(P_{\alpha}\right)+\frac{1}{2} \frac{d}{d T_{S}}\left(P_{\xi} \tanh (\xi)\right)\right] ; \quad d T_{s}=\frac{d \eta}{2 S(\eta)}
$$

The wave function of this system can be decomposed over the eigenfunctions of the Kazimir operator with the eigenvalues $\mathcal{K}_{\epsilon}$

$$
\begin{gathered}
\Psi\left(T_{s}=T_{s}(T)-T_{s}(0) \mid \alpha\right)=\sum_{\epsilon}\left[A_{\epsilon}^{(+)} e^{+\imath \mathcal{K}_{\epsilon} T_{s}} \Psi_{\epsilon}\left(\alpha_{T} \mid \alpha_{0}\right)+A_{\epsilon}^{(-)} e^{-\imath \mathcal{K}_{\epsilon} T_{s}} \Psi_{\epsilon}^{*}\left(\alpha_{T} \mid \alpha_{0}\right)\right], \\
\Psi_{\epsilon}\left(\alpha_{T} \mid \alpha_{0}\right)=Y_{S O(4,1)}\left(\alpha_{T}\right) Y_{S O(4,1)}^{*}\left(\alpha_{0}\right) \exp \left\{\imath P \xi\left(\tanh \left(\xi_{T}\right)-\tanh \left(\xi_{0}\right)\right)\right\},
\end{gathered}
$$

where $A^{( \pm)}$are the operators of the creation and annihilation of the Universe, $Y_{S O(4,1)}$ is a unitary irreducible representation of the $S O(4,1)$ group. This wave function reproduces the physical picture of the Misner anisotropic Universe (16) discussed above in section 4.

In the large time limit, the $S O(4,1)$ symmetry is broken, the Kazimir operator term disappears in comparison with the mass term. In this case, the masses of elementary particles in the Weinberg-Salam model are determined by the fixed values of angles of the scale-scalar field mixing and the ADM-observer gets the Friedmann cosmological models of radiation and dust, considered above. 


\section{Conclusion}

We have shown that including the time surface term in the canonical Hamiltonian formulation of GR helps us to extract the time-like variable and its conjugated momentum from the extended phase space. By taking into account the time surface term we represented here the new version of the Dirac - ADM Hamiltonian formalism for general relativity in the reduced phase space with the Schrödinger - like equation for a wave function describing the quantum evolution of the Universe. This evolution coincides with the Friedmann classical evolution of the dust filled Universe and shows that in GR like in special relativity there are two distinguished invariant time variables: the "phase time" of the ADM-observer (who constructs the Hamiltonian and measures the time as a phase of the wave function of the expanding Universe ) and the geometrical time of the Friedmann observer (who measures the time as an invariant proper interval and observes this expansion on the earth).

In special relativity, the corresponding times are connected by the Lorentz transformation and they coincide only in the case when the rest frame of the Einstein observer coincides with the rest frame of a particle. Now the main question is to find the corresponding transformation from the rest frame of the ADM - observer to the Friedmann one.

\section{Acknowledgment}

The authors thank Profs. A. Ashtekar, B M. Barbashov, G.T. Horowits, V.G. Kadyshevsky, K. Kuchar, D.A.Kirzhnitz, L.N. Lipatov, D.V.Volkov for useful discussions. One of the authors (V.N.P.) acknowledges the hospitality of the International Centre for Theoretical Physics in Trieste where this paper was finished.

\section{References}

[1] P.A.M. Dirac. Proc.Roy.Soc., A 246 (1958) 333; Phys.Rev. 114 (1959) 924.

[2] R. Arnowitt, S. Deser, C.W. Misner. Phys.Rev. 117 (1960) 1595.

[3] J.A.Wheeler. In Batelle Recontres : 1967 Lectures in Mathematics and Physics, edited by C. DeWitt and J.A.Wheeler, Benjamin, New York, (1968).

[4] B.S. DeWitt. Phys.Rev. 160 (1967) 1113.

[5] C. Misner. Phys.Rev. 186 (1969) 1319.

[6] M.P. Ryan, Jr., and L.C. Shapley. "Homogeneous Relativistic Cosmologies", Princeton Series on Physics, Princeton University Press, Princeton, N.Y. 1975;

M.P. Ryan, "Hamiltonian Cosmology", Lecture Notes in Physics N 13 Springer Verlag, Berlin-Heidelberg-New York, 1972. 
[7] K. Kuchar. Journ.of Math.Phys. 17 (1976) 777.

[8] A.L. Zelmanov. Dokl.AN USSR 107 N 6 (1956) 315;

Dokl. AN USSR 209, N 4 (1973) 822.

[9] D.Hilbert, Nachrichten K. Gesellschaft. Wiss. Göttingen. Math.-phys.Klasse, 3 (1915) 395.

[10] T. Regge, C.Teitelboim. Annals of Phys. N.Y. 88 (1974) 286.

[11] V.N.Pervushin. JINR Rapid Commun. 6/57/-92, 46 (1992); V.N.Pervushin, T.Towmasjan. J.Moscow Phys.Soc. 3, 1 (1993); V.N.Pervushin, V.V.Papoyan in "Symmetry methods in Physics " edited A.N.Sissakian. Dubna, (1994) V.2, 396.

[12] A.M.Khvedelidze, V.V.Papoyan, V.N.Pervushin. Phys.Rev.D 51, (1995) 5654.

[13] V.I.Smirichinskii. On the Hamiltonian reduction in the Ashtekar variables. (be published)

[14] B.A. Dubrovin, S.P. Novikov, A.T. Fomenko. Modern Geometry - Methods and Applications, Part II, The Geometry and Topology of Manifold (Springer Verlag, New York, 1984); D.M. Gitman, I.V. Tyutin, Quantization of Fields With Constraints (Springer Verlag, Bonn, 1990).

[15] S.A.Gogilidze,A.M.Khvedelidze, V.N.Pervushin. On the ADM formulation of general relativity with the time surface term. (be published)

[16] R.Penrous. Relativity, groups and topology. N.Y.-Lond. Gordon and Breach, 1964.

[17] N.A.Chernicov, E.A.Tagirov. Ann. Inst. H. Poincare 1968 V.9A. p.109-141.

[18] Jacob D.Bekenstein. Ann.of Physics Vol. 82 Feb. 1974 N2 p.535. 